\title{
Toward Reliable Intra-Body Molecular Communication: An Error Control Perspective
}

\author{
Ligia F. Borges, Michael T. Barros, and Michele Nogueira
}

\begin{abstract}
The authors contribute to a
detailed and precise view of

existing methods for error control

in in-body molecular communi-

cation. They classify the free-dif-

fusion and cell signaling sources

of noises. They present an error

prevention technique, encoding

multiple molecular information

carriers for cell-signaling-based

molecular communication sys-

tems. The article highlights open

research opportunities.
\end{abstract}

\begin{abstract}
Progress in bioengineering and nanotechnology has led to advances in implantable nanoscale devices. Providing synthetic molecular communication among these devices is an ongoing unsolved challenge. Biological systems inspire promising approaches for engineering data communication in molecular communication, but noises from dynamic tissue signaling result in low data rate and high latency. Thus, error control techniques become critical for reliable communication. This article contributes to a detailed and precise view of existing methods for error control in in-body molecular communication. It classifies the free-diffusion and cell signaling sources of noises. The article presents an error prevention technique, encoding multiple molecular information carriers for cell-signaling-based molecular communication systems. It highlights open research opportunities.
\end{abstract}

\section{INTRODUCTION}

Molecular communication (MC) has extraordinary potential to transform medical technology. Leveraging on advances in nanotechnology, MC allows implantable nanomachines to communicate by biological principles for data transmission. Nanomachines are devices less than 100 $\mu \mathrm{m}$ in size with primitive sensing, actuating, and computing capabilities. A nanonetwork arises from the communication among these devices and operates inside the human body assisting on different fronts, such as intelligent drug delivery, infectious agent detection, and cancer cell identification [1]. MC has various healthcare applications once it allows the controlled actuation of nanomachines inside bodies and living tissues, and the integration between a nanonetwork and the Internet.

Reliable MC is essential in healthcare applications. It depends on multiple building blocks to achieve a complete communication system, such as efficient information coding and error control. The MC research community has investigated a range of biologically inspired methods and the use of molecules to engineer in-body data communication. The preference for MC rather than the traditional electromagnetic waves occurs due to $M C^{\prime}$ s increased biocompatibility and higher energy efficiency [2]. Researchers adopt distinct elements of biochemical communication processes and abstract them into models for assembling and characterizing systems for encoding information and controlling errors in data transmission.

Small particles act as chemical signals conveying the information. A synthetic transmitter nanomachine releases these particles into a fluidic medium, where the particles propagate until they arrive at a receiver nanomachine, which detects and decodes the information encoded in the particles. This process is prone to different errors introduced by the network entities and channel properties in which noises are inevitable, hindering the development of reliable MC. Reliable and error-tolerant MC techniques are vital for nanomedicine applications. Communication errors may lead to the erroneous delivery of particles to incorrect body parts, which can cause unprecedented and harmful effects to the human body.

Based on a deep search of the MC literature, we observe only initial discussions about error control and specific noises in different diffusion-based MC systems and variants thereof. Even though some studies point to interesting and tractable solutions, they are specific to some cases and do not necessarily translate to various MC systems. For example, some studies focus on bionano machine architectures, and modulation and coding techniques [3], while other studies are specific solutions, such as congestion control [4], as well as inter-symbol interference (ISI) reduction channel codes [5].

Given the importance of error control for designing reliable $M C$, this article promotes a structured and focused discussion on this topic, aiming to lead research on error control for MC. Besides providing a discussion on how the community addresses the error control issue for intrabody MC systems, it presents a new multi-carrier solution based on the abundantly available molecules in tissues and organs. Molecules, such as $\mathrm{Ca}^{2+}, \mathrm{IP}_{3}$, and $\mathrm{K}^{+}$, and their nonlinear relationships are explored to control error with a multi-molecule encoding mechanism and parity checks. Simulation results show improvements in capacity and reduction in path loss.

This article proceeds as follows. The following section provides a brief background on MC and the impact of noise within it. We then present an overview of the sources of errors in MC. Following that, we classify existing error control techniques in the literature. We then present the proposed encoding technique for error prevention in cell-signaling-based MC. Finally, we highlight open issues and then conclude the article. 


\begin{tabular}{|c|c|c|c|c|c|}
\hline Type & Propagation & Example & Channel & Speed & Range \\
\hline Intracrine & Free diffusion & $\begin{array}{l}\text { Stem cells, Prokaryote, } \\
\text { Eukaryote cells }\end{array}$ & Intracellular space & $18-60,000 \mathrm{~nm} / \mathrm{s}$ & $0.5 \mu \mathrm{m}-20 \mu \mathrm{m}$ \\
\hline Autocrine & Reaction-diffusion & $\begin{array}{l}\text { Stem cells, Prokaryote, } \\
\text { Eukaryote cells }\end{array}$ & Tissue & $18-60,000 \mathrm{~nm} / \mathrm{s}$ & $0.5 \mu \mathrm{m}-60 \mu \mathrm{m}$ \\
\hline Juxtacrine & Reaction-diffusion & $\begin{array}{l}\text { Stem cells, Prokaryote, } \\
\text { Eukaryote cells }\end{array}$ & Gap junction & $18-60,000 \mathrm{~nm} / \mathrm{s}$ & $0.5 \mu \mathrm{m}-400 \mu \mathrm{m}$ \\
\hline Paracrine & Advection-diffusion & $\begin{array}{l}\text { Stem cells, Prokaryote, } \\
\text { Eukaryote cells }\end{array}$ & $\begin{array}{l}\text { Extracellular, vlood, various } \\
\text { fluids, free space }\end{array}$ & $18 \mathrm{~nm} / \mathrm{s}-120 \mathrm{~cm} / \mathrm{s}$ & $0.5 \mu \mathrm{m}-2 \mathrm{~m}$ \\
\hline Endocrine & Advection-diffusion & Eukaryote cells & Circulatory system & $120 \mathrm{~cm} / \mathrm{s}$ & $0.5 \mu \mathrm{m}-2 \mathrm{~m}$ \\
\hline
\end{tabular}

TABLE 1. Compilation of characteristics of different cellular signaling types $[3,7,8]$.

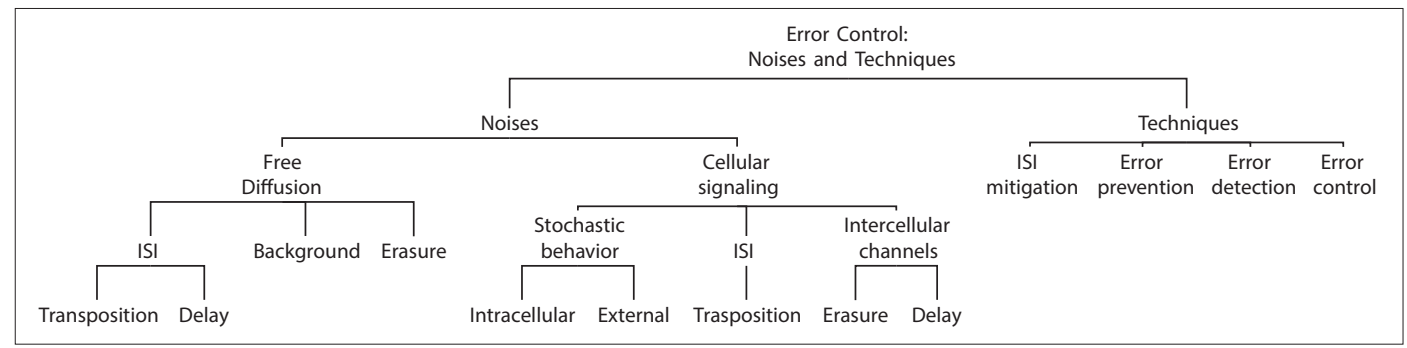

FIGURE 1. Classification of error control: types of noises and techniques.

\section{MoleCULAR COMMUNICATIONS AND THE IMPACT OF NOISES}

Molecular communication noise must be handled based on the characterization of environmental dynamics, as stochastic chemical reactions govern it. The effects caused by the stochastic behavior inherent in chemical reactions are the main source of error [6]. There are a variety of molecules, proteins, amino acids, and other elements that react with each other. The dynamic behavior increases with the number of active cells in tiny tissue volumes. This results in different signaling known to biology and biochemistry as intracrine, autocrine, juxtacrine, paracrine, and endocrine signals.

Intracrine signals produced by the receiver cell stay within it. Autocrine signals are produced by receiver cells and affect the receiver cell itself. Juxtacrine signals are from adjacent (touching) cells. These signals are transmitted along cell membranes via protein or lipid components integral to the membrane and affect either the emitting cell or cells immediately adjacent. Paracrine signals receive information in the vicinity of the transmitter cell (e.g., neurotransmitters); signaling cells secrete signal molecules into the extracellular fluid. Endocrine signals receive information from distant transmitting cells. Endocrine cells produce hormones that travel through the blood to reach all parts of the body. Table 1 presents metrics associating all these types of cellular signaling for communications, including cells, propagation type, channel, signaling wave speed, and communication range.

Nanomachines cannot implement complex coding schemes due to computing limitations, the lack of control at that scale, and their dependence on signaling mechanisms and molecule type. There are two main classes of information coding schemes. One depends on the type of the employed particle (e.g., neurotransmitters, intra- cellular messengers, DNA molecules), and the other relies on the way they employ the particles as means of propagation (e.g., free diffusion and cell-cell signaling). The above-mentioned encoding mechanisms do not tackle the tissue signaling environment. Existing techniques include error prevention, interference mitigation, error detection, and error control codes. Each technique relies on the propagation and the use of particles to encode information.

MC presents challenges, such as the expected computational constraints. The high rate of path loss and noisy transmissions increase the likelihood of communication error. Hence, the design of reliable MC is challenging. Diffusion-based MC enfolds distinct techniques and cellular signaling types, such as free diffusion communication and $\mathrm{Ca}^{2+}$ signaling. Free diffusion follows the behavior of molecules suspended in a fluid that moves in the absence of chemical reactions. $\mathrm{Ca}^{2+}$ signaling lies in a cell-to-cell communication usually mediated by gap junctions after a chemical reaction (i.e., reaction-diffusion). Different types of signaling might have a different number of chemical reactions due to more or fewer information carriers, which makes each signaling scheme have its own propagation characteristics (Table 1).

\section{A CLASSIFICATION OF NOISES AND THEIR SOURCES}

Figure 1 presents a classification of the most prominent noises in MC (detailed in this section) and the main techniques to handle these noises and errors resulting from them (detailed later). Noises in MC follow two different perspectives: diffusion-related and signaling-related noises.

\section{FreE-DIFFUSION-RELATED NoISES}

The randomness from the movement of the information particles is one of the main sources of noise in free-diffusion-based MC (Fig. 2). ISI emerges from these noises, dramatically affecting communication reliability. ISI results from the 


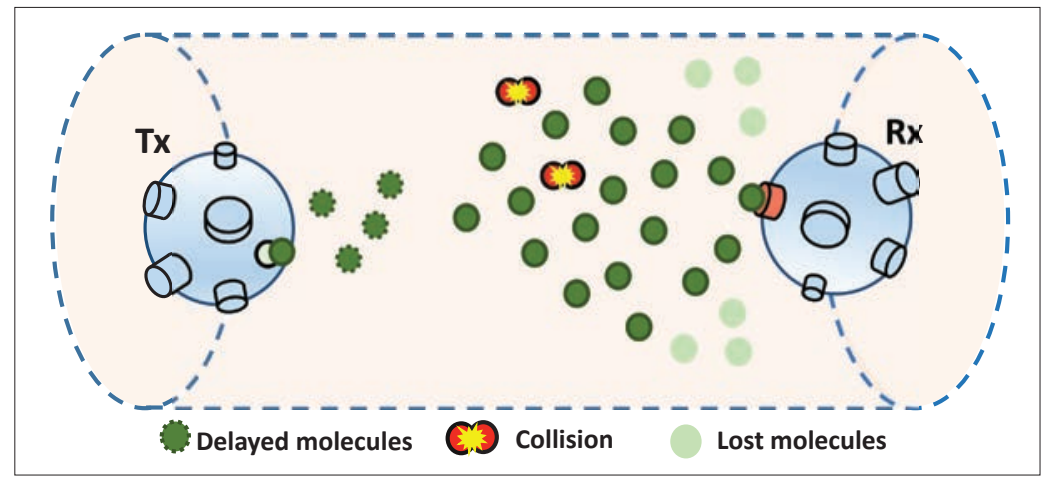

FIGURE 2. Representation of a free-diffusion-based MC system and noises.

delayed arrivals of information particles or arrivals out of order, which generate transposition among symbols (i.e., transposition noise, where instead of being distorted, symbols exchange their positions) compromising the detection and decoding of the received signals [8].

Another feature lies in the transmitter-receiver distance as a parameter influencing communication. Depending on the environment (e.g., bloodstream), the nanomachine position may constantly change [9]. When the distance is known, one can identify the probability of erasing noises and optimizing data transmission (e.g., frequency and concentration). An erasure noise consists of particles sent and lost in the diffusion process before reaching the destination. Molecules may be erased by several mechanisms, such as absorption by another receiver or being trapped in biological structures [10].

Noises occur as well with undesired reactions between information molecules and other molecules present in the environment (e.g., collisions breaking particles or generating new molecules). These reactions change the structure of the information molecules, and therefore the receiver cannot detect the transmitted message (decomposition noise). The background noise or biological interference is generated by molecules other than those employed for communication, which shows a similar affinity for the receptors [11].

\section{CeLLULAR SIGNALING-Related NoISES}

Molecular emission for cellular communication (e.g., $\mathrm{Ca}^{2+}$ signaling) promotes noisy conditions that produce an error in data transmission. In cellular signaling systems, the transmitting and receiving nanomachines are cells (synthetic, hybrid, or natural). Synthetic biology provides tools to control, modify, and reengineer the cells' genetic code to change their structure and functions [1]. As propagation happens through molecular transportation from one cell to another, depending on the type of cell, it may be controlled by communication gates. Thus, noise is concentrated on these gates and the characteristics of the cells. Gap junctions are gates opening and closing between the cytoplasm of two neighbor cells. Each cell connects to several other cells through these gates, and as they have stochastic behavior, diffusion turns to cells that are not the destination cells, generating losses (erasure noise). In this communication, ISI occurs by delayed molecules in the event of an unexpected gate close during transmission, or in large cells that affect the veloci- ty of the molecule propagation due to the amount of space or also with symbol transposition.

Molecular signaling might result in excessive noise due to variations in molecular concentrations during intercellular (i.e., between-cell) signaling (Fig. 3). In cell-cell signaling, both internal and external noise may occur. Internal noise happens due to the constant fluctuations in molecular concentrations within cells, given the stochastic events of chemical reactions. External noise results from the permeability of intercellular channels, and it is generated by fluctuations in molecular concentration coming from neighboring cells [12].

\section{ERROR CONTROL TECHNIQUES}

Conventional systems apply complex error control techniques to ensure reliable communication. Because of the particular constraints to MC, these techniques cannot be directly adapted to nanonetworks. This section presents different techniques in MC to detect, mitigate, and correct errors in communication channels. It follows a four-like classification for these techniques (Fig. 1), including:

- ISI mitigation

- Error prevention techniques

- Error detection codes

- Error control codes

\section{InTER-SYMBOL INTERFERENCE MItIGATION}

ISI mitigation aims to increase channel reliability by reducing the effects of the stochastic nature of diffusion. Solutions follow longer symbol duration or reduced molecular concentration by lower energy symbols. Modulation and encoding schemes for ISI mitigation keep low complexity. An example is to use modulation schemes based on particle release. The reduction of the transposition error information results from three main approaches:

- Counting the number of released particles or alternating two different particles in two subsequent time slots [8]

- Sending the information in two types of molecule, one for symbol-1 and another for symbol-0

- Sending two different molecules at the same time, subtracting their concentration and identifying the equalized signal [8]

These techniques require precise discrimination among different symbols by the receiver.

There are also ISI-resistant coding schemes for free diffusion systems. This approach mitigates delay in the arrival of particles based on the number of received molecules. However, there is a time-gap among consecutive symbols [5]. These mitigation schemes require either devices able to perform simple tasks, such as molecule storage or production, sensing, and accounting, or additional resources to control the release. These nanomachines can be generated by artificial cells that combine biological and synthetic structural elements, or genetically modified cells through synthetic biology, where specific functionalities can be integrated into the cells [2].

\section{ERror Prevention TeCHnIQUES}

Error prevention needs to be tailored to the different MC channels. Channel impulse response $(\mathrm{CIR})$ is an expressive characteristic for the chan- 
nel estimates. MC systems analyze CIR through the expected number of molecules in the receiver. The receiver estimates the transmitter distance through path loss of CIR. Knowing this distance is paramount to reduce the channel error probability, because the transmitter optimizes transmission properties, for example, longer pulses or increased signal amplitude for longer distances to minimize erasure noise effects [3]. CIR in MC changes over time because nanomachines are not fixed in their position [8], rendering precise CIR estimation difficult and useless [3]. Also, this technique might require multiple transmissions, and it greatly occupies the channel. Another approach to estimating the distance lies in measuring molecule propagation time based on the propagation delay metric. The receiver requests a feedback signal, and after receiving the response, it estimates the distance based on the propagation delay. This technique requires increased computational power from the transmitter.

\section{ERror Detection Codes}

Proposals for free-diffusion-based systems have employed the parity check method for error detection. One approach lies in sending concentrations of two distinguishable molecule types using a modulated parity check encoder. In [10], the information is encoded into a binary code (i.e., representing the two states of the cell) characterized by the codeword of three symbols. Its decoder computes the a posteriori log-likelihood ratio of the information, symbols to identify possible errors. This proposal uses concepts from synthetic biology to design a cell with branching genetic circuits (i.e., a network of chemical reactions involving genes and molecular species working together) to encode and modulate codeword symbols.

Similarly, the Hamming code shows low complexity, which is an advantage for MC. However, there is an energy cost when applied with numerous random number generators and for encoding and decoding processes. Some derivations of the Hamming code have been considered for MC. For example, the Hamming distance designs channel codes based on the number of different symbols between two binary sequences. Hamming distance combined with channel timing-based decoding is effective to combat additive noise sources in non-concentration-encoded MC channels [10]. However, it is not well suited to combat erasure errors.

The Hamming-weight codes determine path lengths between nodes and use the weight of a string to detect and reduce transposition errors. LT erasure codes and the Hamming code (LT-Hamming) combat the information loss in molecular-scarce (e.g., diffusive channels with erasure noises) communication scenarios. These techniques rely on a random number of generators that consumes computational resource and energy [10]. They allow error detection, but not error correction.

\section{ERror Correction Codes}

Error correction codes aim at reducing bit error rate. There are two different techniques: forward error correction (FEC) and backward error correction (BEC). FEC often employs an error con-

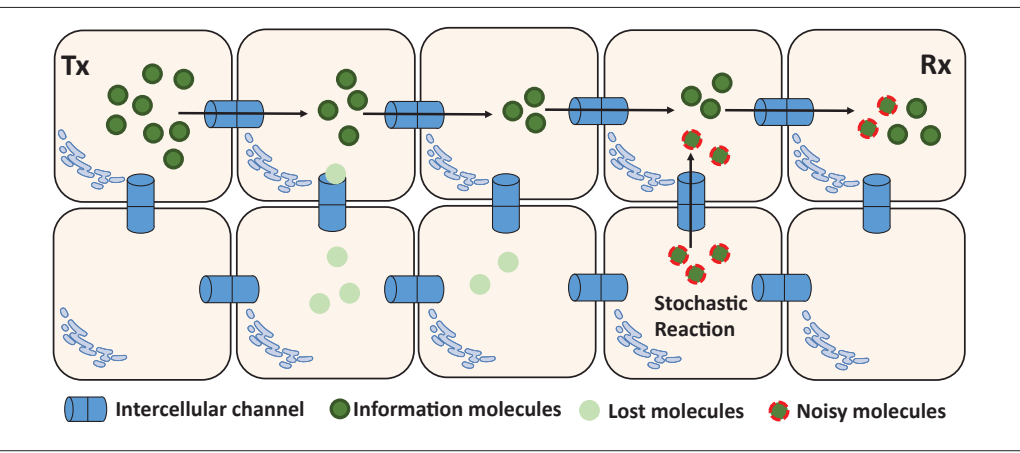

FIGURE 3. Representation of a cellular signaling-based MC system and noises.

trol coding (e.g., Hamming codes and Reed Solomon). Due to the expected limitations of nanoscale devices, considering computational complexity and energy-efficient communication is paramount. Minimum energy codes (MECs) with Hamming distance constraints are an option to reduce energy consumption by lowering the average weight of codewords in diffusion-based MC. Reducing the average code weight decreases the average codeword weight energy. Thus, applying MECs combined with on-off keying (OOK) modulation (transmitting molecules in symbol-1 periods and not transmitting molecules in symbol-0 periods) is suitable for diffusive systems, because transmitting the symbol-0 requires no energy. MECs, as channel code, improve performance but require large codeword lengths. They outperform the Hamming codes in bit error rate generated by ISI [13]. However, the complexity of coding systems must remain low to allow applications. Among the most potent techniques concerning error correction are codes based on linear factors, such as Reed Solomon code, but they require high energy consumption.

$B E C$ techniques exist for $M C$, such as stopand-wait automatic repeat request (SW-ARQ). Some proposals apply the SW-ARQ method in free diffusion systems considering the restrictions of computation and memory in nanomachines. Variations of this technique send duplicated messages and acknowledgment (ACK) to improve reliability. According to the technique, the ACK control message performs multiple tasks, such as triggering the next information in the transmitter, stopping and releasing information molecules, and changing the type of molecule [4]. Feedback-based schemes such as SW-ARQ control the flow and ensure delivering a given number of information particles to the receiver while preventing the transmitter from releasing molecules faster than the receiver can react. This avoids molecules remaining in the channel, colliding with each other, or diffusing away, causing loss or ISI, but it yields additional time cost and energy consumption. Each technique has its limitations and advantages, and the choice must consider the application.

\section{ERror Prevention TeChNIQUE FOR A CELL-SIGNALING-BASED MC SYSTEM}

The natural signaling process of the human body cells has inspired promising approaches for engineering data communication in MC. An example 


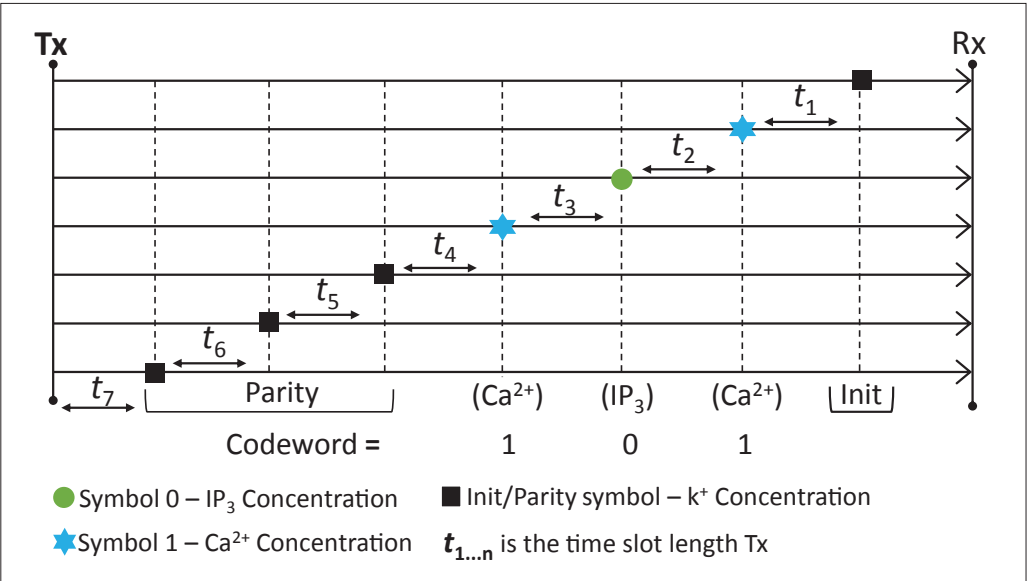

FIGURE 4. Error prevention technique. decoding the received signals. Tx decides which molecule to send depending on the previously sent transmission symbol and current symbol. However, it must satisfy the size of the codeword length $I \leq 7$ symbols (i.e., $I=3$ message symbols, $I \leq 3$ parity check symbols, and $I=1$ symbol for a start signal). A time slot $(t)$ is reserved for the release of each symbol (i.e., symbol time interval) based on the propagation time. The natural mechanism of the cells causes the molecules to arrive through the intercellular channels to be quickly expelled from the cells; then, even if the time for release is added, communication tends to be fast.

Given the asynchronous communication and the difficulty of feedback signals, the proposed scheme considers a single symbol of $\mathrm{K}^{+}$at the beginning of the codeword to indicate a new transmission. Error detection is performed using a parity check scheme. The parity symbols are added in the last positions of a string of the binary code and comprise a concentration of $\mathrm{K}^{+}$. The parity symbol ensures that the total number of symbols- 1 in the string is even if three consecutive $\mathrm{K}^{+}$symbols are included at the end of the codeword or odd if only one symbol is added (Fig. 4). For example, to transmit a 3-symbol message 101, the transmitter sends the $\mathrm{Ca}^{2+}$ molecules to symbol- 1 and then releases $\mathrm{IP}_{3}$ to the symbol-0 and $\mathrm{Ca}^{2+}$ for the next symbol. Here, parity adds three $\mathrm{K}^{+}$symbols at the end of the codeword to indicate that the total number of ones (i.e., $\mathrm{Ca}^{2+}$ ) in the string is even. The receiver nanomachine recognizes that when $\mathrm{IP}_{3}$ or $\mathrm{Ca}^{2+}$ are followed by $\mathrm{K}^{+}$, it indicates the parity symbol. Once all messages begin with $\mathrm{K}^{+}$and there are no other cases where $\mathrm{IP}_{3}$ or $\mathrm{Ca}^{2+}$ are followed by $\mathrm{K}^{+}$, the parity symbol also indicates the end of the message. The same message is repeated at least three times with a long period of the interval $T_{b}$ between each repetition. Thus, the receiver checks parity and discards error messages.

Based on [12], the proposed error prevention technique has been studied through mathematical simulations for a cellular-signaling-based MC system. We assume an asynchronous single-hop MC system composed of a hybrid transmitter and receiver nanomachine (i.e., a biological nanodevice with electronic parts) and channel. Rx measures the concentration of molecules upon contact and checks the parity symbol. Cell-tocell communication occurs when the gap junction in the cells opens, and $\mathrm{IP}_{3}, \mathrm{Ca}^{2+}$, and $\mathrm{K}^{+}$ molecules propagate through the cytosol. In this investigation, intracellular noises have been modeled for $\mathrm{IP}_{3}$ and $\mathrm{Ca}^{2+}$ based on [14]. Figure 5 compares the proposed technique with OOK modulation to transmitting both molecules in symbol-1 periods and not transmitting molecules in symbol-0 [12].

The comparison follows a perspective of information theory. In MC, molecules may not arrive at the receiver due to their diffusion direction probability in intercellular channels. Thus, path loss was applied to analyze this behavior using different molecules as information carriers, considering the intra/intercellular channel noises. Shannon's entropy quantifies the communication capacity in terms of symbols considering a discrete memoryless channel, as proposed in [15]. 
We define the state transition probabilities for the $\mathrm{Rx}$ and $\mathrm{Tx}$. For $\mathrm{Tx}$, we consider the release of $\mathrm{Ca}^{2+}$ or $\mathrm{IP}_{3}$ molecules. For the receiver, we consider another two states to represent when the amount of received molecules (i.e., $\mathrm{Ca}^{2+} / \mathrm{IP}_{3}$ ) changes cell state, transitioning from symbol- 1 and symbol-0. The mutual information measures the quantity of transferred information.

Results demonstrate that end-to-end capacity improves up to 10 percent when information encoding uses multiple information carriers in combination with the proposed error prevention (EP) technique. The intracellular noise causes communication degradation, and the error prevention technique reduces path loss, only by creating a dual signaling mechanism using different carrier molecules, which has not been greatly utilized before. Results pave the way for more in-depth studies into how many molecules can be used in an interwoven manner to maximize communication performance in MC.

\section{OPEN ISSUES AND OPPORTUNITIES}

Despite advances in MC, many challenges exist for the design of reliable communication. In reaction-diffusion channels, such as biological tissues, there are different sources of noise and errors (Fig. 1). Thus, it is unclear whether conventional techniques comply with MC requirements. This section presents the main open issues and opportunities for research on error control in MC in biological tissues.

Although the literature explores nano communication models, there are few proposals to deal with noises and errors. In cellular signaling, one of the main areas for future contribution lies in developing tailored error prevention techniques considering the signaling factors that affect coding such as concentration amplitude and transmission time. Nanomachines have limited computational capacities, so techniques concerning error correction (e.g., Reed-Muller and feedback-based techniques) are not suitable. Thus, coding complexity has to be extremely low, and must possibly be implemented through spatial-temporal-based protocols. This is because different tissues present different signaling frequencies as well as spatial dimensions [12].

Another open issue consists of the design of coding schemes that use multiple types of molecules. There are opportunities for characterizing noises caused by different transmitters and receivers. Some studies measure the required energy to achieve the coding technique by metrics such as energy per bit, energy cost to calcium release, bit-meters, and critical distance [13], particularly, for the juxtacrine signaling, or even specific to other types of signaling. Also, the identification of orthogonal waves in signaling molecules (e.g., $\mathrm{Ca}^{2+} / \mathrm{IP}_{3}$ ) is still open.

Natural MC systems have schemes for error control that can inspire the design of new ones. The use of enzymes was proposed to degrade molecules of the channel and reduce ISI effects in free-diffusion-based systems [9]. Similarly, the control of gap junction behavior and organelles, which clean up the cellular environment, can reduce cellular-signaling-related noises and mitigate ISI. Further studies to characterize noise in natural $\mathrm{MC}$ are required to design error control

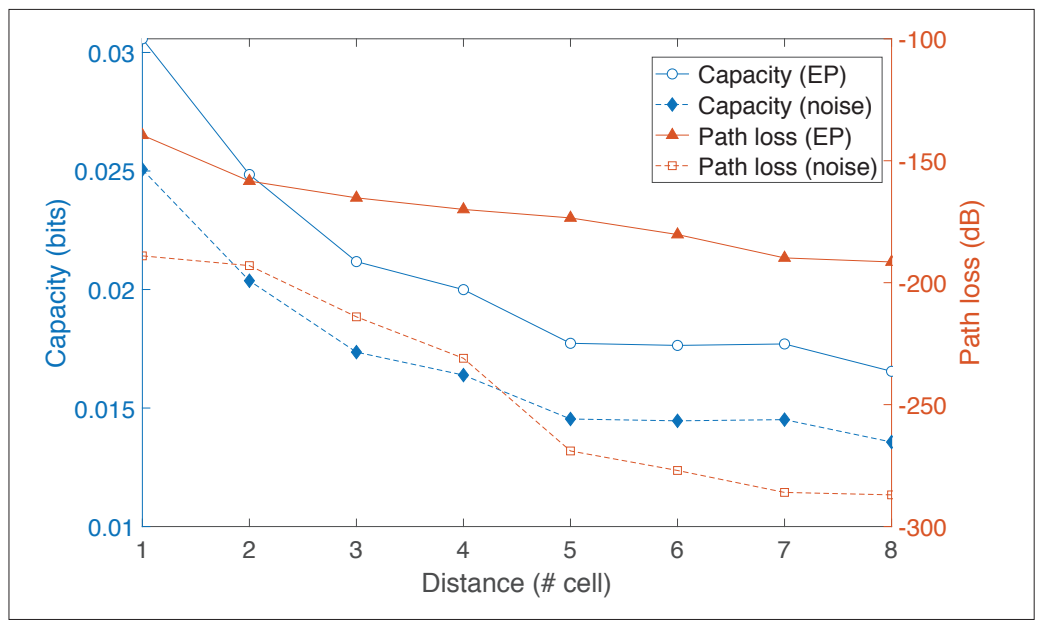

FIGURE 5. Capacity and path loss.

inspired by natural physiological predictive models. Because biological tissues are so complex, more knowledge about these noise sources will elicit novel error control techniques.

Exploiting synthetic biology techniques has value in the design of signaling processing circuits, as suggested by [1], but now focusing on error control (e.g., design synthetic biology components, including encoders, noise filters, and molecule sensing devices). Artificial manipulation has enabled cells to produce engineered molecules, which can serve several approaches, such as developing artificial cell signaling. Although genetic circuit-based and nanomaterials-based error control techniques exist, the implementation of those artificial MC systems should validate their error control capabilities. Similarly, implementing coding schemes that consider the limitations of biological nanomachines remains an open issue, and should take into account experimental data.

\section{CONCLUSION}

Handling noises is critical to advance communication systems once noises significantly compromise communication reliability. The main contributions of this article are fourfold:

- A reinforcement of the importance of addressing noises as primary sources of errors in molecular communication

- A classification of the main types of noises and existing error detection, mitigation, and correction techniques

- The proposal of a new error prevention technique for a cell-signaling-based molecular communication system that has overall superior performance and improves data propagation

- Open issues and opportunities to advance the design of reliable molecular communication.

\section{ACKNOWLEDGMENTS}

This study was financed in part by the Coordenação de Aperfeiçoamento de Pessoal de Nível Superior - Brasil (CAPES) - Grant number 88882.382196/2019-01. M. T. Barros is funded by the European Union's Horizon 2020 research and innovation programme under the Marie Skłodowska-Curie grant agreement No 839553. 


\section{REFERENCES}

[1] I. F. Akyildiz et al., "The Internet of Bio-Nano Things," IEEE Commun. Mag., vol. 53, no. 3, Mar. 2015, pp. 32-40.

[2] I. F. Akyildiz, M. Pierobon, and S. Balasubramaniam, "Moving Forward with Molecular Communication: From Theory to Human Health Applications," Proc. IEEE, vol. 107, no. 5, 2019, pp. 858-65.

[3] M. Kuscu et al., "Transmitter and Receiver Architectures for Molecular Communications: A Survey on Physical Design with Modulation, Coding, and Detection Techniques," Proc. IEEE, vol. 107, no. 7, 2019, pp. 1302-41.

[4] L. Felicetti, M. Femminella, and G. Reali, "Congestion Control in Molecular Cyber-Physical Systems," IEEE Access, vol. 5, 2017, pp. 10,000-11.

[5] A. Keshavarz-Haddad, A. Jamshidi, and P. Akhkandi, "Inter-Symbol Interference Reduction Channel Codes Based on Time Gap in Diffusionbased Molecular Communications," Nano Commun. Networks, vol. 19, 2019, pp. 14856.

[6] T. Nakano et al., "Random Cell Motion Enhances the Capacity of Cell-Cell Communication," IEEE Trans. Molecular, Biological and Multi-Scale Commun., vol. 5, no. 2, 2019, pp. 158-62.

[7] R. A. Bradshaw and E. A. Dennis, Handbook of Cell Signaling, Academic Press, 2009.

[8] V. Jamali et al., "Channel Modeling for Diffusive Molecular Communication - A Tutorial Review," Proc. IEEE, vol. 107, no. 7, 2019, pp. 1256-1301.

[9] S. R. Rouzegar and U. Spagnolini, "Diffusive Mimo Molecular Communications: Channel Estimation, Equalization, and Detection," IEEE Trans. Commun., vol. 67, no. 7, 2019, pp. 4872-84.

[10] Z. Wei et al., "Hamming-Luby Rateless Codes for Molecular Erasure Channels," Nano Commun. Networks, vol. 23, 2020, p. 100,280 .

[11] A. Marcone, M. Pierobon, and M. Magarini, "Parity-Check Coding Based on Genetic Circuits for Engineered Molecular Communication Between Biological Cells," IEEE Trans. Commun., vol. 66, no. 12, 2018, pp. 6221-36.

[12] M. T. Barros, S. Balasubramaniam, and B. Jennings, "Comparative End-to-End Analysis of Ca 2+-Signaling-Based Molecular Communication in Biological Tissues," IEEE Trans. Commun., vol. 63, no. 12, 2015, pp. 5128-42.

[13] Y. Lu, M. D. Higgins, and M. S. Leeson, "Comparison of Channel Coding Schemes for Molecular Communications Systems," IEEE Trans. Commun., vol. 63, no. 11, 2015, pp. 3991-4001.
[14] G. Yu et al., "A Constructive Role of Internal Noise on Coherence Resonance Induced by External Noise in a Calcium Oscillation System," Chaos, Solitons \& Fractals, vol. 41, no. 1, 2009, pp. 273-83.

[15] I. F. Akyildiz et al., "An Information Theoretic Framework to Analyze Molecular Communication Systems Based on Statistical Mechanics," Proc. IEEE, vol. 107, no. 7, 2019, pp. 1230-55.

\section{BIOGRAPHIES}

LIGIA F. BORGES is a Ph.D. candidate in computer science at Federal University of Paraná, Brazil. She received her M.Sc. degree in computational technologies for agribusiness from Federal University of Technology, Paraná, and her B.Tech. degree in computer networks from the Higher Education Centre at Foz do Iguaçu, Brazil. Her main research interests include nanonetworks, molecular communications, and bio-inspired techniques.

Michael Taynnan Barros is a lecturer with the School of Computer Science and Electronic Engineering, University of Essex United Kingdom, and the recipient of the Marie Skłodowska-Curie Individual Fellowship (MSCA-IF) at the BioMediTech Institute of Tampere University, Finland. He received his Ph.D. in telecommunication software from the Waterford Institute of Technology, Ireland, in 2016. He has authored or co-authored over 60 research papers in various international flagship journals and conferences in the areas of wireless communications, molecular and nanoscale communications, as well as bionanoscience. $\mathrm{He}$ is also a reviewer for many journals and has participated as a Technical Program Committee member and reviewer for various international conferences. Research interests include the Internet of BioNanoThings, molecular communications, bionanoscience, and 6G communications.

MiCHeLE NOGUeIRA [SM] is an associate professor in the Computer Science Department at Federal University of Paraná. She received her doctorate in computer science from Sorbonne University, UPMC, LIP6, France (2009), and she was on a sabbatical leave at Carnegie Mellon University (2016-2017). Her research interests include wireless networks, network security, and dependability. Her works search to provide resilience to self-organized, cognitive, and wireless networks by adaptive and opportunistic approaches. She was one of the pioneers in addressing survivability issues in self-organized wireless networks. She has served as an Associate Technical Editor for IEEE Communications Magazine and the Journal of Network and Systems Management. She also serves as Chair for the IEEE ComSoc Internet Technical Committee, and she is a Senior Member of ACM. 